\title{
MAIN CAUSES OF PAIN AND OTHER VERTEBRAL
}

SYNDROMES

Petro Kolisnyk ${ }^{1}$, Serhii Kolisnyk ${ }^{1}$, Rostyslav Kravets ${ }^{1}$, Ihor Humeniuk ${ }^{1}$

${ }^{1}$ Department of Medical Rehabilitation and Medical Social Medicine, National Pirogov Memorial Medical University, Vinnytsya, Ukraine, 21018

Introduction.

Vertebral pain may occur for many reasons and complicates diagnostics, treatment, rehabilitation.

It must be taken into consideration the spectrum of pathomorphological changes in vertebral segments that can lead to lesion of the segmental nervous and vascular structures (SNVS).

We propose to define these pathanatomical changes as traumatic elements.

Purpose is to determine the role of main traumatic elements (TE) in pain and other vertebral syndromes occurrence

\section{Method.}

We carried out X-ray and MRI screenings of 234 patients with vertebral pain or other syndromes.

Considering the placing of SNVS, X-ray and $M R I$ scans were analyzed to identify the main TE.

Results

TE in Degenerative Disk Disease: vertebral subluxations - 65,8\%, osteophytes of vertebral bodies and facets - 75,6\%, foraminal stenosis - 93,2\%. Disk herniation $16.67 \%$, among which hernial bulging in the direction of segmental nervous structures was diagnosed only in $6.25 \%$ of cases. The role of disc herniation as TE is exaggerated, as evidenced by other authors. Significantly, the role of TE can be performed by swelling of facet joints' capsules (56.25\%).

Spondyloarthrosis: facet's deformities $86.5 \%$, foraminal stenosis - 50,7\%, osteophytes of facets $-76,5 \%$.
Osteoporosis: deformities of vertebral bodies $99,6 \%$, deformation of articular and uncinate processes - 94,2\%, postcompressive displacement of vertebral body mass - $26,5 \%$, foraminal stenosis - $98,7 \%$, vertebral subluxations were diagnosed in $20.2 \%$ of cases.

Spondylosis, ligamentosis: ligamental ossifications - $86.8 \%$, foraminal stenosis $80.9 \%$

Signs of segmental nervous structures irritation were diagnosed in $83.3 \%$, compression - in $16.7 \%$ of patients.
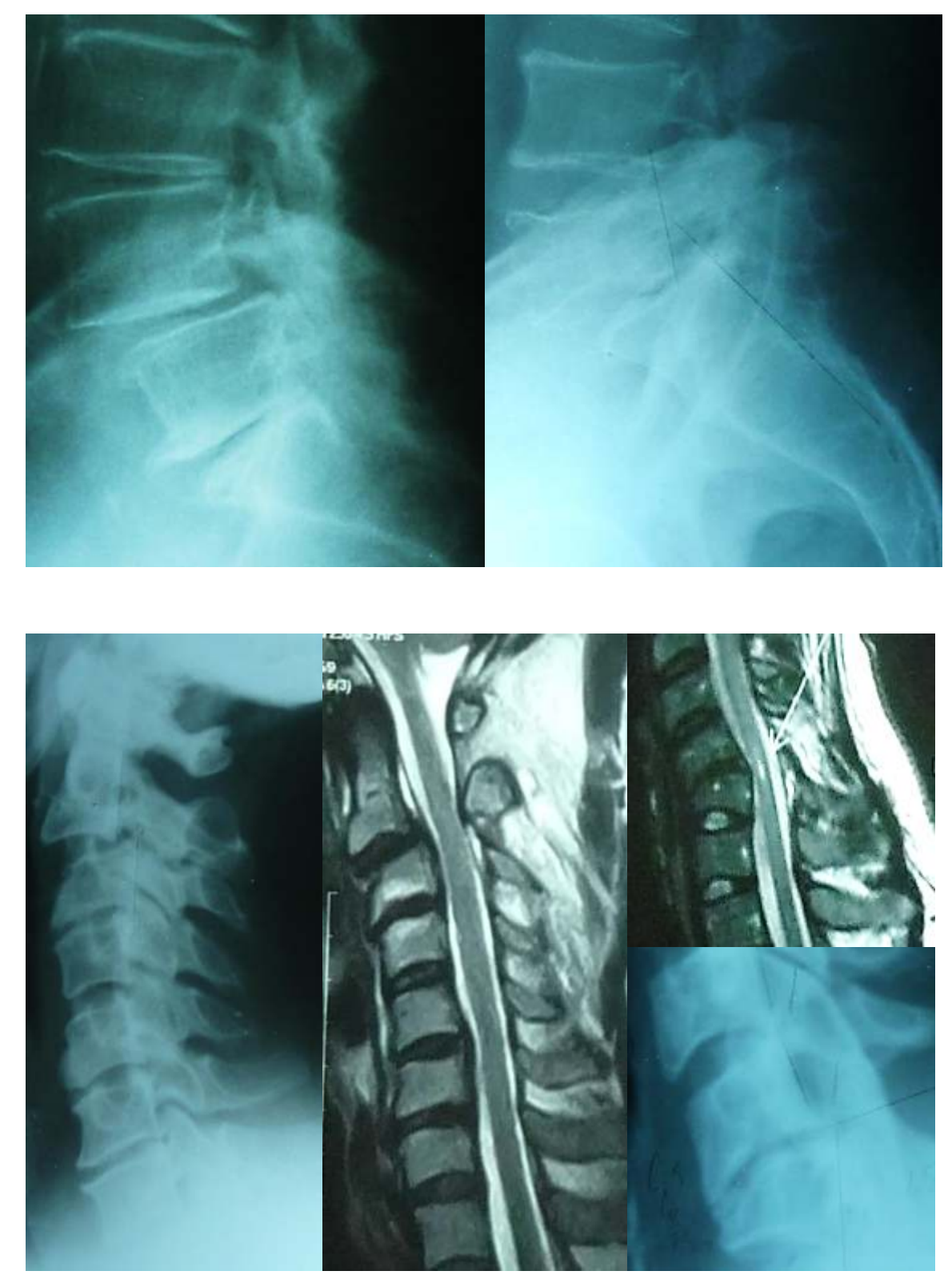

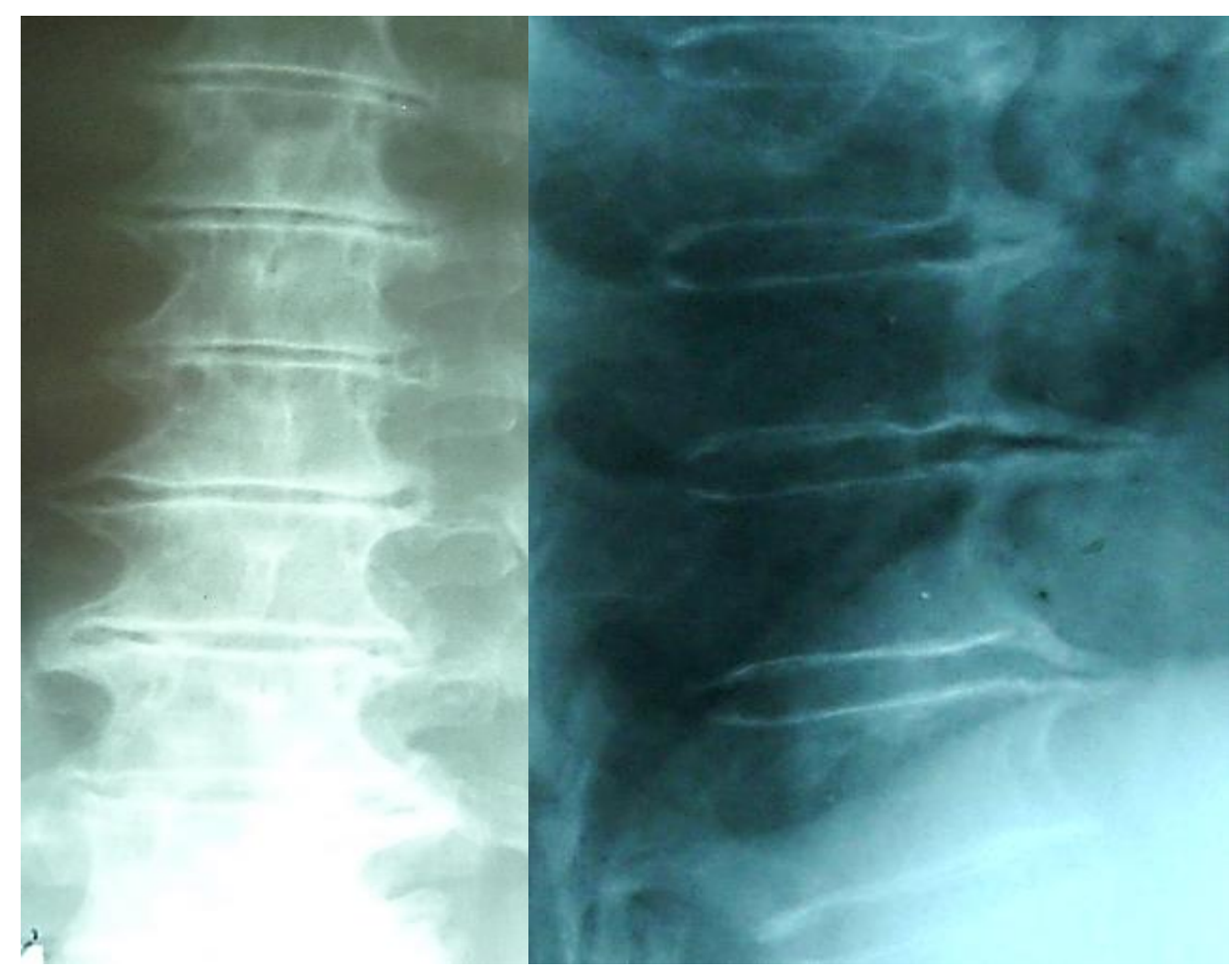

Conclusions

1. Pathanatomic changes directed towards the SNVS may act as TE.

2. Severity of clinical manifestation of spine pathology depends on the degree of raumatization of segmental nervous or vascular structures more, than on nosology.

3. By X-ray and MRI changes, it's impossible to differentiate traumatic elements on irritating or compressing ones.

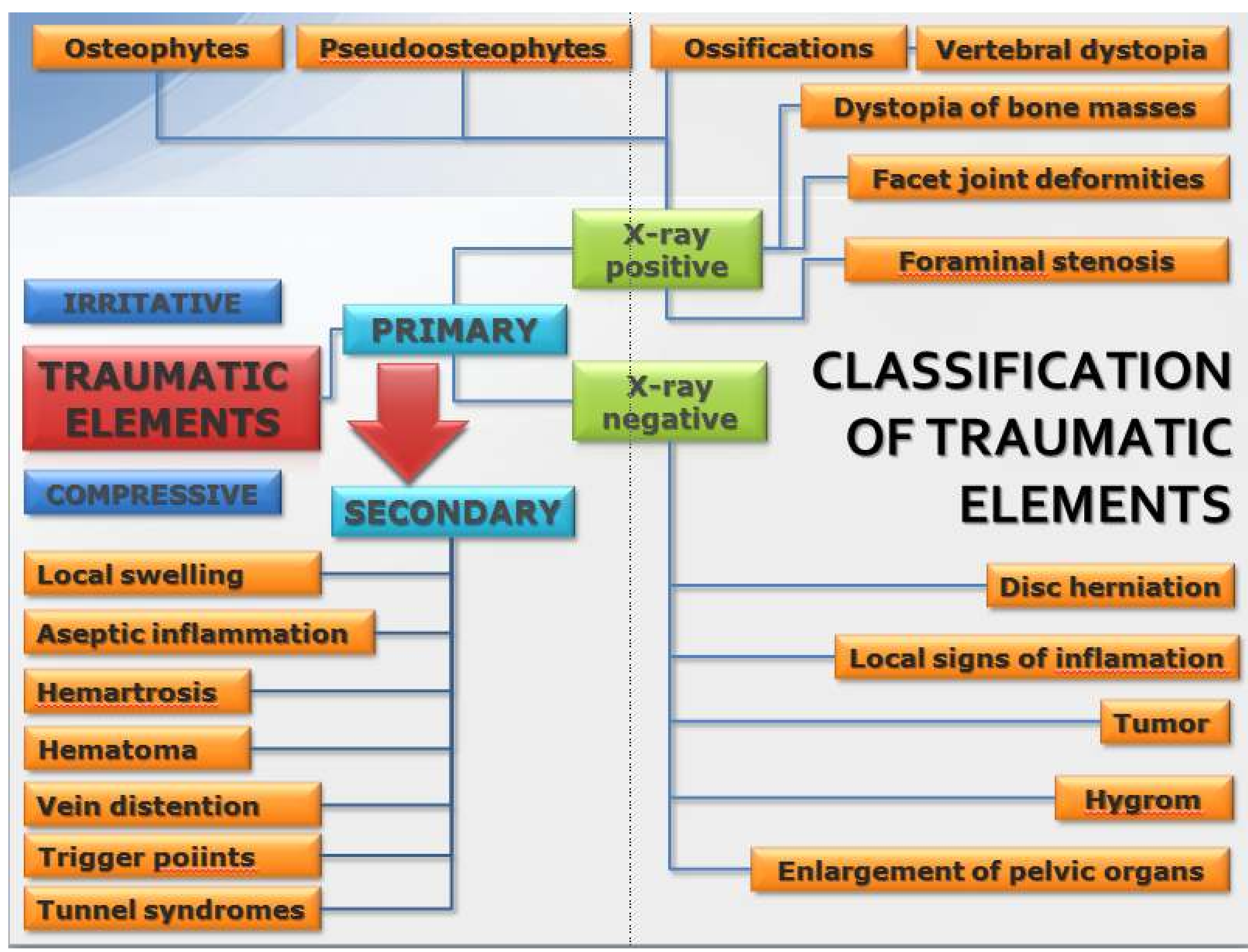

